\title{
New Approach of Homotopy Perturbation Method for Solving the Equations in Enzyme Biochemical Systems
}

\author{
Kurunatha Perumal Thevar Vijayan Preethi ${ }^{1}$, Rajaram Poovazhaki ${ }^{1}$, Lakshmanan Rajendran ${ }^{2,}$ * \\ ${ }^{1}$ Department of Mathematics, E. M. G. Yadava Women’s College, Madurai, Tamilnadu, India \\ ${ }^{2}$ Department of Mathematics, Sethu Institute of Technology, Kariyapatty, Tamilnadu, India
}

Email address:

rain.preethi90@gmail.com (K. P. T. V. Preethi), rpoovazhaki@yahoo.co.in (R. Poovazhaki), raj_sms@rediffmail.com (L. Rajendran)

${ }^{*}$ Corresponding author

\section{To cite this article:}

Kurunatha Perumal Thevar Vijayan Preethi, Rajaram Poovazhaki, Lakshmanan Rajendran. . New Approach of Homotopy Perturbation Method for Solving the Equations in Enzyme Biochemical Systems. Applied and Computational Mathematics.

Vol. 6, No. 3, 2017, pp. 161-166. doi: 10.11648/j.acm.20170603.14

Received: March 31, 2017; Accepted: April 17, 2017; Published: June 27, 2017

\begin{abstract}
In this paper, new homotopy perturbation method (iteration scheme) will be employed to solve the nonlinear dynamical problems that arise in thin membrane kinetics. More precisely, the method will be used to mathematically model and solve the kinetics of the thin membrane. A main property that makes the proposed method superior to other iterative methods is the way it handles boundary value problems, where both mixed Dirichlet and Neumann boundary conditions are taken into consideration, while other iterative methods only make account of the initial point and as a result, the approximate solution may deteriorate for values that are far away from the initial point and closer to the other endpoint. Our analytical results are compared with numerical solution. The method is found to be easily implemented, fast, and computationally economical and attractive.
\end{abstract}

Keywords: Mathematical Modelling, Thin Membrane, Enzyme Kinetics, Homotopy Perturbation Method

\section{Introduction}

Non-linear differential equations can model many phenomena in different fields of science and engineering in order to present their behaviors and effects by mathematical concepts. Most of the equations do not have analytical solution which can be handled by semi-analytical or numerical methods. In order to obtain exact solution of nonlinear differential equations, semi-analytical methods such as the Variational Iteration method (VIM) and Homotopy perturbation method (HPM) are considered. The ideas of the VIM and HPM were first pioneered by He [1,2]. He [3] presented application of the HPM in solving the nonlinear non-homogeneous partial differential equations. They are powerful algorithms in solving various kinds of linear and nonlinear equations. The homotopy perturbation method is used by Nourazar et al. [4-6] in order to obtain exact solution of nonlinear differential equations.. Abzari and Abzari [7] presented numerical study of Burgers-Huxley equations via reduced differential transform method.
Rajendran and co-workers are solved many non-linear equation in chemical science using homotopy perturbation method and variational iteration method [8]. Comparative analysis of variational iteration method and Haar wavelet method for the numerical solutions of Burgers-Huxley and Huxley equations is presented by Ray and Gupta [9]. Soori et al. $[10,11]$ presented application of the variational iteration method and the 2 homotopy perturbation method to the fisher type equation. Also, Soori [12] presented series solution of weakly-singular kernel volterra integro-differential equations by the combined laplace-adomian method. Application of the variational iteration method for the Newell-Whitehead-Segel equation is presented by Soori [13]. Also recently Malini et al. [14] compared variational iteration, NHPM and ADM method with numerical solution for non-linear equation in single valued carbon nanotubes. In this study we have applied NHPM to find the approximate solution of the problem of the second order non-linear differential equation in thin membrane. This method generate the analytical solutions in convergence series and it is also effective mathematical tool to handle a large class of linear and non- 
linear differential equation in engineering and chemical sciences.

\section{Mathematical Formulation of the Problem}

In enzyme biochemical systems kinetics in thin membrane is modelled by the reaction-diffusion equations [1]:

$$
\begin{gathered}
\frac{d^{2} u}{d x^{2}}-\lambda u v-u w=0 \\
\frac{d^{2} v}{d x^{2}}-\lambda u v=0 \\
\frac{d^{2} w}{d x^{2}}-u w+\lambda u v=0
\end{gathered}
$$

where $u(\mathrm{x}), v(\mathrm{x})$ and $w(\mathrm{x})$ denote the concentrations of the chemical species, $\mathrm{A}, \mathrm{B}$ and $\mathrm{C}$ respectively. The diffusion coefficient of three species is considered to have an equal diffusion coefficient which is equal to 1 . Now the boundary conditions becomes

$$
\begin{array}{r}
x=0, u=\alpha, \frac{d v}{d x}=0, w=\gamma \\
x=1, \frac{d u}{d x}=0, v=\beta, \frac{d w}{d x}=0,
\end{array}
$$

\section{NOMENCLATURE}

Symbols
$u$
$v$
$w$
$\lambda$
$\alpha$
$\beta$
$\gamma$

$$
\begin{aligned}
& \text { Name } \\
& \text { Concentrations of species A } \\
& \text { Concentrations of species B } \\
& \text { Concentrations of species C } \\
& \text { React ration constant } \\
& \text { Concentrations of A at } x=0 \\
& \text { Concentrations of B at } x=1 \\
& \text { Concentrations of C at } x=0
\end{aligned}
$$

\section{Solution of Boundary Value Problem Using HPM}

Recently, many authors have applied the HPM to various problems and demonstrated the efficiency of the HPM to hand non-linear structure and solve various physics and engineering problems [5-7]. This method is a combination of homotopy in topology and classic perturbation techniques. Ji-Huan He used the HPM to solve non-linear boundary value problems [11]. These wide varieties of applications show the power of the HPM in solving functional equations. The HPM is unique in its applicability, accuracy and efficiency. The HPM [13] uses the imbedding parameter $\mathrm{p}$ as a small parameter and only little alteration is needed to search for an asymptotic solution. Recently, Eswari et al [15], derived the approximate analytical expressions of concentration and current in homogeneous catalytic reactions at spherical microelectrodes using the homotopy perturbation method. Thiagarajan et al. [16] presented the approximate analytical expressions for steadystate catalytic current of mediated bioelectrocatalys is using the homotopy perturbation method. The basic concept of homotopy perturbation method is given in Appendix A. Solving the above equation (1) to (3) using new homotopy perturbation method (Appendix B) for the boundary conditions (4) and (5), we get concentration of species as follows:

$$
\begin{gathered}
u(x)=\frac{\alpha}{\cosh (\sqrt{\lambda \beta+\gamma})} \cosh (\sqrt{\lambda \beta+\gamma}(x-1)) \\
v(x)=\frac{\beta}{\cosh (\sqrt{\lambda \alpha})} \cosh (\sqrt{\lambda \alpha} x) \\
w(x)=\left[\frac{(\gamma-\lambda \beta)}{\cosh (\sqrt{\alpha})} \cosh (\sqrt{\alpha}(x-1))\right]+\lambda \beta
\end{gathered}
$$

\section{Numerical Simulation}

The non-linear differential equations (1-3) are solved by numerical methods. The functionpdex 4 in SCILAB software which is a function of solving the boundary value problems for differential equation is used to solve this equation. Its numerical solution is compared with homotopy perturbation method in Figures1-3 and it gives a satisfactory result for various values of parameter. The MATLAB program is also given in Appendix B.

\section{Discussions}

Fig. 1 shows the dimensionless steady-state concentration of the species A. using Eq. (6) for various values of dimensionless parameter $\lambda$. From these figures, it is inferred that the value of the species A increases gradually when $\lambda$ decreases. From Fig. 2, it is observed that the value of the concentration of species $\mathrm{B}$ is also increases when $\lambda$ decreases. Concentration of species A and B is uniform when $\lambda \leq 0.001$. Fig. 3 shows the dimensionless steady-state concentrations of species C using Eq. (8). From this figure it is observed that concentration of species $\mathrm{C}$ decreases when $\lambda$ increases.

\section{Conclusion}

A nonlinear time-independent differential equation for in thin membrane was formulated and solved using the HPM. Analytical expressions for the concentrations of species were derived using the HPM. Our approximate analytical results offermore advantages over traditional methods for an efficient operation on thin membrane. The extension of the procedure to other two-dimension and three-dimension geometrics and coupled processes of first-order chemical reactions with complex mixed-boundary conditions seems possible. 


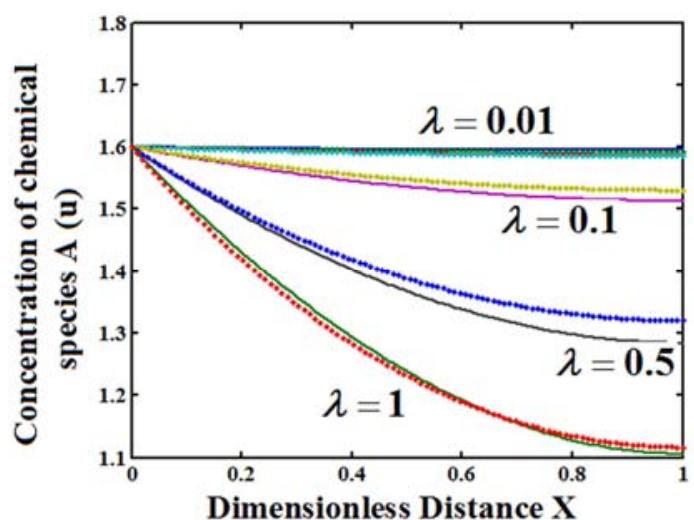

Figure 1. Normalized steady-state concentration of species $A(u)$. The concentrations of species $A$ are computed using eq. (6) for various values of the dimensionless parameter $\lambda$. Value of the other parameters are $\alpha=1.6$, $\beta=0.01$ and $\gamma=0.8$.

(-) represent the equation (6), and (.............) represent the numerical solution.

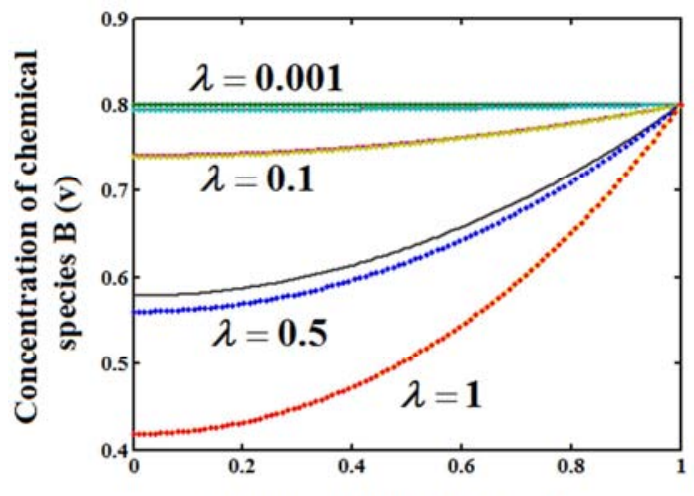

Dimensionless Distance $\mathbf{X}$

(-) represent the equation (7), and (.............) represet the numerical solution.

Figure 2. Normalized steady-state concentration of species $B(v)$. The concentrations of species $B$ are computed using eq. (7) for various values of the dimensionless parameter $\lambda$. Value of the other parameters are $\alpha=1.6$, $\beta=0.01$ and $\gamma=0.8$.

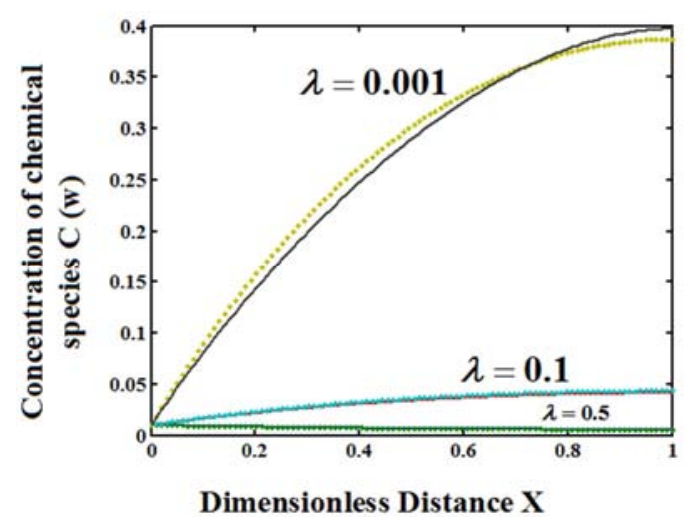

Figure-3. Normalized steady-state concentration of species $C(w)$. The concentrations of species $C$ are computed using eq. (8) for various values of the dimensionless parameter $\lambda$. Value of the other parameters are $\alpha=1.6$, $\beta=0.01$ and $\gamma=0.8$.

(-) represent the equation (8), and (.............) represented the numerical solution.

\section{Appendix-A: Basic Idea of Homotopy Perturbation Method for Initial Value Problem}

We outline the basic idea of Homotopy perturbationmethod. This method has eliminated the limitations ofthe traditional perturbation methods. On the other hand itcan take full advantage of the traditional perturbationtechniques, so there has been a considerable deal of researchin applying homotopy technique for solving variousstrongly non-linear equations. To explain this method,let us consider the following function

$$
A(u)-f(t)=0, \quad t \in \Omega
$$

With the boundary conditions of

$$
B\left(u, \frac{\partial u}{\partial n}\right)=0, \quad t \in \Gamma
$$

where $A, B, f(t)$ and $\Gamma$ denote a general differential operator, a boundary operator, a known analytical function on the domain $\Omega$, respectively. Generally speaking, the operator $A$ can be divided into a linear part $L$ and a non-linear part $N$.

$$
L(u)+N(u)-f(t)=0
$$

By the homotopy technique, we construct a homotopy $v(t, p): \Omega \times[0,1] \rightarrow \Re$, which satisfies:

$$
\begin{aligned}
& H(v, p)=(1-p)\left[L(v)-L\left(u_{0}\right)\right]+p[A(v)-f(t)]=0, \\
& p \in[0,1], t \in \Omega
\end{aligned}
$$

or

$$
H(v, p)=L(v)-L\left(u_{0}\right)+p L\left(u_{0}\right)+p[N(v)-f(t)]=0,
$$

where $p \in[0,1]$ is an embedding parameter, and $u_{0}$ is an initial approximation of Eq. A1, which satisfies the boundary conditions. Obviously from Eqs. A4 and A5, we will have

$$
H(v, 0)=L(v)-L u_{0}(t)=0(\mathrm{~A} 6)
$$

When $p=0$ Eq. A4 or A5 becomes a linear Equation; when $p=1$ it becomes a non-linear Equation. So the changing process of $p$ from zero to unity is just that of

$L(v)-L u_{0}(t)=0$ to $A(v)-f(t)=0$. We can first use the embedding parameter $p$ as a "small parameter", and assume that the solutions of Eqs. A4 and A5 can be written as a power series in $p$

$$
v=v_{0}+p^{1} v_{1}+p^{2} v_{2}+\cdots
$$

Setting $p=1$, results in the approximate solution of Eq. (A1) 


$$
u=\lim _{p \rightarrow 1} v=v_{0}+v_{1}+v_{2}+\cdots
$$

The combination of the perturbation method and the Homotopy method is called the Homotopy perturbation method.

\section{Appendix B: Analytical Solutions of Non-Linear Equations (1)-(3) Using HPM}

In this Appendix, we indicate how the solutions of the equations B1 to B3 are derived.

$$
\begin{gathered}
\frac{d^{2} u}{d x^{2}}-\lambda u v-u w=0 \\
\frac{d^{2} v}{d x^{2}}-\lambda u v=0 \\
\frac{d^{2} w}{d x^{2}}-u w+\lambda u v=0
\end{gathered}
$$

We construct the Homotopy for the above equations as follows:

$$
\begin{gathered}
(1-p)\left[\frac{d^{2} u}{d x^{2}}-\lambda u v(x=1)-u w(x=0)\right]+p\left[\frac{d^{2} u}{d x^{2}}-\lambda u v-u w\right]=0 \\
(1-p)\left[\frac{d^{2} v}{d x^{2}}-\lambda u(x=0) v\right]+p\left[\frac{d^{2} v}{d x^{2}}-\lambda u v\right]=0 \\
(1-p)\left[\frac{d^{2} w}{d x^{2}}-u(x=0) w+\lambda u(x=0) v(x=1)\right]+p\left[\frac{d^{2} w}{d x^{2}}+\lambda u v-u w\right]=0
\end{gathered}
$$

Using the boundary conditions (4) and (5) the above equations becomes as follows:

$$
\begin{gathered}
(1-p)\left[\frac{d^{2} u}{d x^{2}}-u(\lambda \beta+\gamma)\right]+p\left[\frac{d^{2} u}{d x^{2}}-\lambda u v-u w\right]=0 \\
(1-p)\left[\frac{d^{2} v}{d x^{2}}-\lambda \alpha v\right]+p\left[\frac{d^{2} v}{d x^{2}}-\lambda u v\right]=0 \\
(1-p)\left[\frac{d^{2} w}{d x^{2}}-\alpha w+\lambda \alpha \beta\right]+p\left[\frac{d^{2} w}{d x^{2}}+\lambda u v-u w\right]=0
\end{gathered}
$$

The approximate solution of (B1- B3) are,

$$
\begin{gathered}
u=u_{0}+p u_{1}+p^{2} u_{2}+. \\
v=v_{0}+p v_{1}+p^{2} v_{2}+\ldots \\
w=w_{0}+p w_{1}+p^{2} w_{2}+.
\end{gathered}
$$

Subsituting Eq. (B10) to Eq. (B12) into Eq. (B7) to Eq. (B9) we have
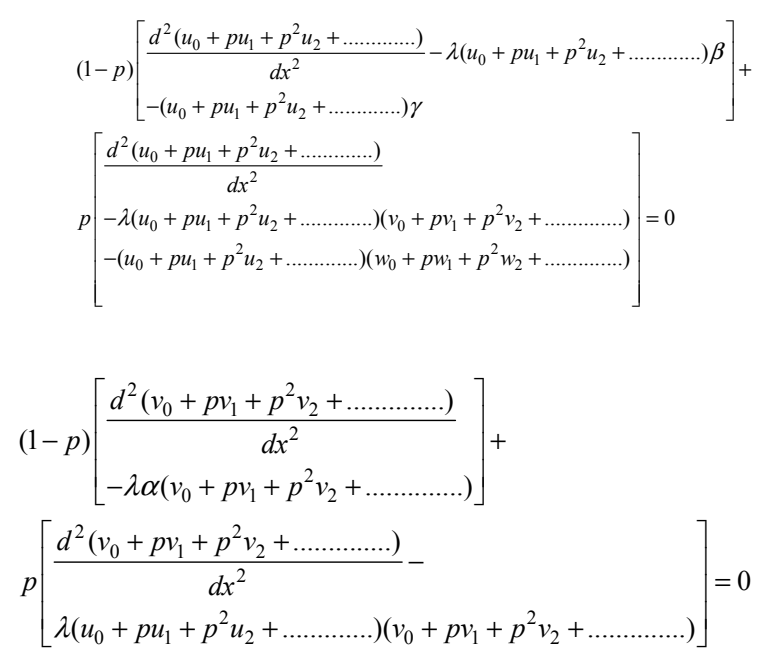

$(1-p)\left[\begin{array}{l}\frac{d^{2}\left(w_{0}+p w_{1}+p^{2} w_{2}+\ldots \ldots \ldots \ldots .\right)}{d x^{2}} \\ -\alpha\left(w_{0}+p w_{1}+p^{2} w_{2}+\ldots \ldots \ldots \ldots . .\right)+\lambda \alpha \beta\end{array}\right]+$

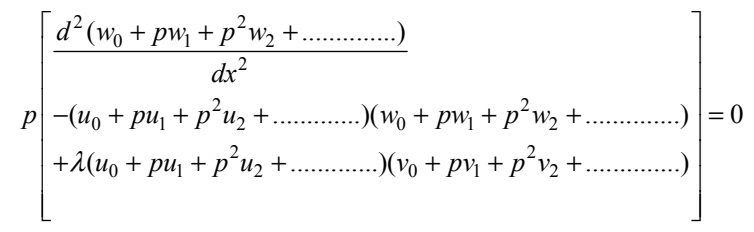

Comparing the coefficients of like powers of $p$ in Eq. (B13) we get

$$
p^{0}: \frac{d^{2} u_{0}}{d x^{2}}-u_{0} \gamma-\lambda u_{0} \beta=0
$$

$p^{1}: \frac{d^{2} u_{1}}{d x^{2}}-\lambda u_{1} \beta-u_{1} \gamma+u_{0} \gamma+\lambda u_{0} \beta-\lambda u_{0} v_{0}-u_{0} v_{0}=0$

Comparing the coefficients of like powers of $p$ in Eq. (B14) we get

$$
\begin{gathered}
p^{0}: \frac{d^{2} v_{0}}{d x^{2}}-\lambda \alpha v_{0}=0 \\
p^{1}: \frac{d^{2} v_{1}}{d x^{2}}-\lambda \alpha v_{1}+\lambda \alpha v_{0}-\lambda u_{0} v_{0}=0
\end{gathered}
$$

Comparing the coefficients of like powers of $p$ in Eq. (B15) we get

$$
\begin{gathered}
p^{0}: \frac{d^{2} w_{0}}{d x^{2}}-\alpha w_{0}+\lambda \alpha \beta=0 \\
p^{1}: \frac{d^{2} w_{1}}{d x^{2}}-\alpha w_{1}+\alpha w_{0}+\lambda u_{0} v_{0}-u_{0} w_{0}-\lambda \alpha \beta=0
\end{gathered}
$$

The initial and boundary conditions are as follows: 


$$
\begin{array}{r}
u_{0}(0)=\alpha \text { and } u_{0, x}(1)=0 \\
u_{i}(0)=\alpha \text { and } u_{1, x}(1)=0, i=1,2, \ldots . \\
v_{0}(0)=\beta \text { and } v_{0, x}(0)=0 \\
v_{i}(1)=0 \text { and } v_{1, x}(0)=0, i=1,2, \ldots . \\
w_{0}(0)=\gamma \text { and } w_{0, x}(1)=0 \\
w_{i}(0)=0 \text { and } w_{i, x}(1)=0
\end{array}
$$

Solving the Eq. (B16) to (B21) and using the boundary conditions (B20) to (B27). We can find the following results:

$$
\begin{gathered}
u_{0}=\frac{\alpha}{\cosh (\sqrt{\lambda \beta+\gamma})} \cosh (\sqrt{(\lambda \beta+\gamma)}(x-1)) \\
v_{0}=\frac{\beta}{\cosh (\sqrt{\lambda \alpha})} \cosh (\sqrt{\lambda \alpha} x) \\
w_{0}=\left[\frac{(\gamma-\lambda \beta)}{\cosh \sqrt{\alpha}} \cosh (\sqrt{\alpha}(x-1))\right]+\lambda \beta \\
u(x) \approx u_{0} \\
v(x) \approx v_{0} \\
w(x) \approx w_{0}
\end{gathered}
$$

Considering the first iteration only we conclude that

Next iteration $\left(2^{\text {nd }}\right.$ (or) $3^{\text {rd }}$ ) will improve the accuracy of the result.

After putting the equations (B31) to (B33) in the equation (B28) to (B30) we obtain the equations $(6)-(8)$ in the text.

\section{Appendix C; Matlab Program to Find the Numerical Solution of Non-linear Equations (1)-(3)}

function ananthu

$\mathrm{m}=0$;

$\mathrm{x}=$ linspace $(0,1)$;

$\mathrm{t}=$ linspace $(0,100000)$;

sol=pdepe(m,@pdex4pde,@pdex4ic,@pdex4bc,x, t);

$\mathrm{u} 1=\operatorname{sol}(:,:, 1)$;

$\mathrm{u} 2=\operatorname{sol}(:,:, 2)$;

u3=sol(:,:,3);

$\%$ figure

$\% \operatorname{plot}(x, u 1(e n d,:))$

$\%$ title('u1 $(\mathrm{x}, \mathrm{t})$ ')

$\% x$ label('Distance $\mathrm{x}$ ')

$\%$ ylabel('u1(x, 2)')

$\%$

$\%$ figure

$\%$ plot(x, u2(end,:))

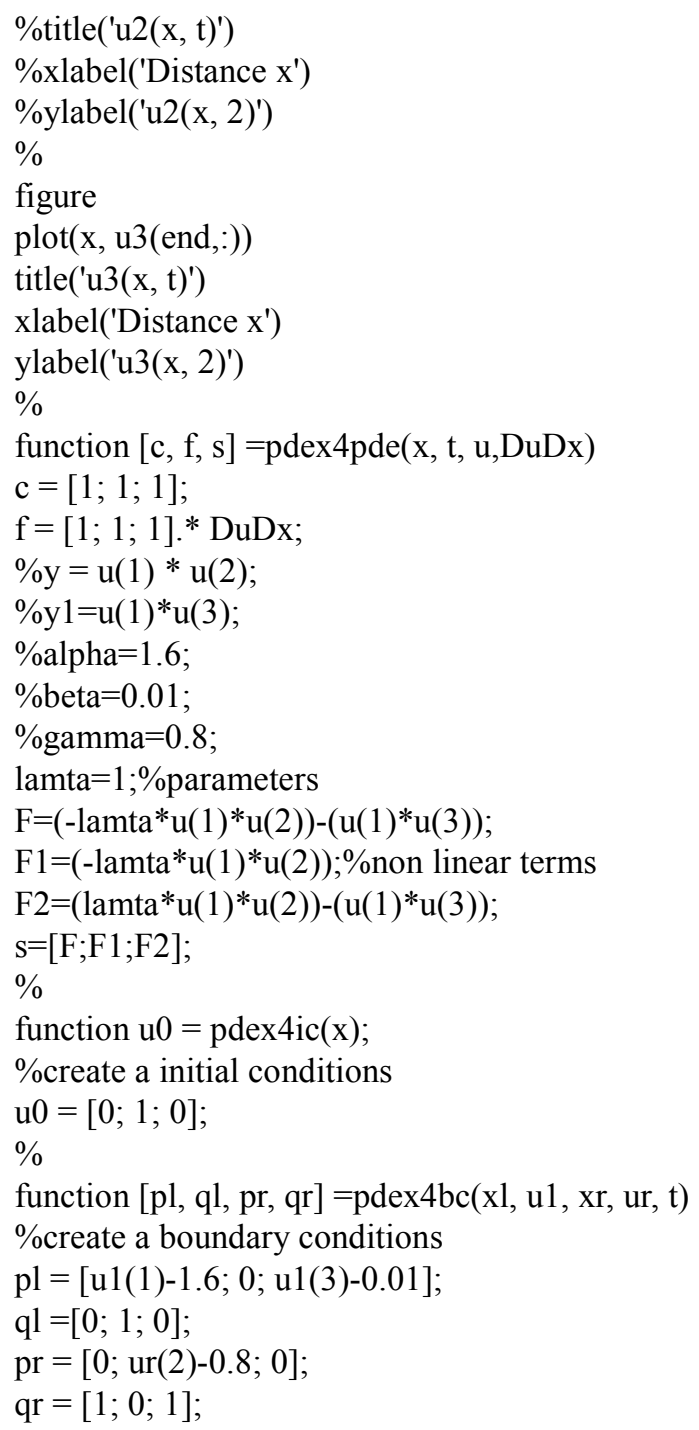

\section{References}

[1] He J. H., "Variational iteration method: A kind of nonlinear analytical technique" Some examples, Int. J. NonLinear Mech., 34(4), 699-708 (1999).

[2] He J. H., "Homotopy perturbation technique", Comput Methods Appl. Mech. Engng., 178(3), 257-262 (1999).

[3] He J. H., "Application of homotopy perturbation method to nonlinear wave equations", Chaos Solit. Fract., 26(3), 695700 (2005).

[4] S. S. Nourazar, M. Soori and A. Nazari-Golshan, "On the exact solution of Newell-Whitehead-Segel equation using the homotopy perturbation method", Aust. J. Basic Appl. Sci., 5(8), 1400-1411(2011).

[5] S. S. Nourazar, M. Soori and A. Nazari-Golshan, "On the exact solution of Burgers-Huxley equation using the homotopy perturbation method", J. Appl. Math. Phy., 3(3), 285-294 (2015).

[6] S. S. Nourazar, M. Soori and A. Nazari-Golshan, "On the homotopy perturbation method for the exact solution of Fitzhugh-Nagumo equation", Int. J. Math. Computation, 27(1), 32-43 (2015). 
[7] R. Abazari and M. Abazari, " Numerical study of BurgersHuxley equations via reduced differential transform method", Computat. Appl. Math., 32(1), 1-17 (2013).

[8] J. Saranya, L. Rajendran, L. Wang,C. Fernandez, "A new mathematical modelling using homotopy perturbation method to solve nonlinear equations in enzymatic glucose fuel cells", Chemical Physics Letters, 317-326,662(2016).

[9] S. S. Ray and A. K. Gupta, "Comparative analysis of variational iteration method and Haar wavelet method for the numerical solutions of Burgers-Huxley and Huxley equations", J. Math. Chemistry, 52(4), 1066-1080 (2014).

[10] S. S. Nourazar, M. Soori and A. Nazari-Golshan, "Application of the variational iteration method and the homotopy perturbation method to the Fisher Type Equation", Int. J. Math. Computation, 27(3), 1-9 (2015).

[11] M. Soori, "The homotopy perturbation method and the variational iteration method to nonlinear differential equations", (2011).

[12] M. Soori, "Series solution of weakly-singular kernel volterra integro-differential equations by the combined laplaceadomian method", (2016).

[13] M. Soori, "The variational iteration method for the NewellWhitehead-Segel equation”, (2016).

[14] R. MaliniDevi, S. Pavithra, R. Saravanakumar, L. Rajendran, "Analysis of nonlinear vibrations of single walled carbon nanotubes", International Journal of Modern Engineering Research (IJMER), 2249-6645, 6(11)(2016).

[15] A. Eswari, L. Rajendran, "Analyticalexpressions of concentration and current in homogeneous catalytic reactions at spherical microelectrodes: Homotopy Perturbation approach", Journal of Electroanalytical Chemistry, 173-184, 651(2011).

[16] S. Thiagarajan A. Meena S. Anitha, L. Rajendran, "Analytical expression of the steady-state catalytic current of mediated bioelectro catalysis and the application of He's Homotopy perturbation method", J Math Chem, 96-104, 6(2) 2011.

[17] Abbasbandy and Elyas Shivanian "Application of variational iteration method for nth-order integro-differential equations", Zeitschrift für Naturforschung A 64 (7-8), 439-444

[18] Saeid Abbasbandy and Elyas Shivanian, "Application of the variational iteration method for system of nonlinear Volterra's integro-differential equations", Mathematical and computational applications 14 (2), 147-158.

[19] Hossein Vosughi and Elyas Shivanian, "A new analytical technique to solve Volterra's integral equations, Mathematical methods in the applied sciences" 34 (10), 1243-1253.

[20] S. Abbasbandy, E. Magyari, E. Shivanian, "The homotopy analysis method for multiple solutions of nonlinear boundary value problems", Communications in Nonlinear Science and Numerical Simulation 14 (9), 3530-3536.

[21] Saeid Abbasbandy and Elyas Shivanian, "Prediction of multiplicity of solutions of nonlinear boundary value problems: novel application of homotopy analysis method", Communications in Nonlinear Science and Numerical Simulation 15 (12), 3830-3846.

[22] Saeid Abbasbandy and Elyas Shivanian, "Solution of singular linear vibrational BVPs by the homotopy analysis method", Journal of Numerical Mathematics and Stochastics 1 (1), 7784.

[23] L. Ahmad Soltani's, Elyas Shivanian, Reza Ezzati, "Convection-radiation heat transfer in solar heat exchangers filled with a porous medium: exact and shooting homotopy analysis solution", Applied Thermal Engineering 103, 537542.

[24] Elyas Shivanian and S. J. Hosseini Ghoncheh, "A new branch solution for the nonlinear fin problem with temperaturedependent thermal conductivity and heat transfer coefficient", The European Physical Journal Plus 132 (2), 97. 